Résumés des conférences et travaux

\title{
Paléographie latine (livres et documents)
}

\section{Olivier Legendre}

\section{(2) OpenEdition \\ 12 Journals}

Édition électronique

URL : https://journals.openedition.org/ashp/535

DOI : 10.4000/ashp.535

ISSN : 1969-6310

Éditeur

Publications de l'École Pratique des Hautes Études

\section{Édition imprimée}

Date de publication : 1 octobre 2008

Pagination : 143-143

ISSN : 0766-0677

\section{Référence électronique}

Olivier Legendre, «Paléographie latine (livres et documents) », Annuaire de l'École pratique des hautes études (EPHE), Section des sciences historiques et philologiques [En ligne], 139 | 2008, mis en ligne le 24 novembre 2008, consulté le 12 juillet 2021. URL : http://journals.openedition.org/ashp/535; DOI : https://doi.org/10.4000/ashp.535 


\title{
PALÉOGRAPHIE LATINE (LIVRES ET DOCUMENTS)
}

\author{
Chargé de conférences : M. Olivier LegEndre
}

Programme de l'année 2006-2007 : Paléographie latine (livres et documents).

Ce cours ayant avant tout un objectif pratique, il est dans sa nature de s'adapter à la demande directe des étudiants. Il a donc été demandé aux auditeurs, lors de la première séance, d'indiquer le type de documents ou la période à laquelle ils s'intéressaient, afin de plier, autant que possible, le choix des transcriptions à leurs préoccupations. La première séance (20 novembre) a en outre été consacrée à un exposé général rapide sur l'histoire de l'écriture, permettant de mettre en place quelques définitions et notions fondamentales et de présenter les instruments de travail essentiels (dictionnaire d'abréviations, manuels, instruments d'identification, exercices disponibles sur Internet, etc.). Quelques exercices de transcription relativement faciles ont enfin permis d'évaluer le niveau des étudiants (Laon, bibl. mun. 407, correspondance d'Hincmar de Reims [IX $\left.{ }^{\mathrm{e}} \mathrm{s}.\right]$; actes de la fin du XIII ${ }^{\mathrm{e}} \mathrm{s}$. : fonds du musée des archives départementales, $\mathrm{n}^{\text {os }} 104$ et 109 [en français]).

Si des actes ou des ouvrages en français ont pu être incidemment abordés à plusieurs reprises au cours de la seconde heure (notamment un extrait du cartulaire de Saint-Riquier [ms. Abbeville, bibl. mun. 854]), l'essentiel des documents transcrits étaient latins. La traduction a donc été systématiquement effectuée au fur et à mesure de la lecture pour confirmer la validité de la transcription et parvenir à une réelle compréhension du texte déchiffré. La priorité a été donnée, tout au long de l'année, aux documents présentant des difficultés significatives par la sévérité ou la fréquence des abréviations, ou par leur caractère cursif, d'où le recours fréquent, quand il s'agissait de manuscrits, à des ouvrages d'exécution sommaire. On s'est fréquemment appuyé aussi sur des manuscrits liturgiques : ceux-ci, extrêmement courants, sont souvent très elliptiques dans leur rédaction, ce qui pose à la fois des problèmes de lecture et d'interprétation, alors même que leur écriture est rarement difficile à déchiffrer. Les références liturgiques étant aussi très présentes dans la datation des actes, on a porté un effort particulier sur le problème de l'identification des pièces liturgiques et de la datation que l'on peut en extraire. Un dernier enjeu important étant de ne pas séparer hermétiquement les écritures livresques et diplomatiques, on a utilisé beaucoup de documents intermédiaires tels que les cartulaires.

Les heures ont été partagées de façon à peu près égale entre le déchiffrage des écritures livresques et de celles des actes, en suivant, tout au long de l'année, une double progression chronologique : la première heure était consacrée aux hautes époques (du $\mathrm{IX}^{\mathrm{e}}$ au XIII ${ }^{\mathrm{e}}$ siècle), la seconde au bas Moyen Âge et au début de la période moderne $\left(\mathrm{XIII}^{\mathrm{e}}-\mathrm{XVI}^{\mathrm{e}}\right.$ siècles). Les écritures antérieures au XII ${ }^{\mathrm{e}} \mathrm{S}$. ont été vues très rapidement 
(acte de Théotolon, archevêque de Tours [EPHE, fonds du musée des Archives départementales, $\mathrm{n}^{\mathrm{o}} 12 \mathrm{]}$; ms. Laon, bibl. mun. 81 : ouvrage d'exégèse, $\mathrm{IX}^{\mathrm{e}} \mathrm{s}$.) afin d'aborder sans tarder les périodes traitées par la majorité des étudiants. Les écritures du XII ${ }^{\mathrm{e}}$ siècle ont fait l'objet de trois séances (janvier-février : exercices fondés notamment sur des actes des arch. dép. d'Eure et Loir et du chartrier de Pontfraud [voir ci-dessous la présentation générale de ce dossier], un manuscrit hagiographique [Laon, bibl. mun. $270^{\text {bis}}$ ] et une bible glosée [Laon, bibl. mun. 71]). Ces séances, portant généralement sur des écritures faciles à lire, ont permis de se concentrer sur la compréhension et la mémorisation des principes du système d'abréviation médiéval. Le XIII ${ }^{\mathrm{e}}$ siècle, permettant d'aborder des genres d'écritures plus difficiles et plus divers, a été traité avec une attention particulière ( 5 séances), autant pour les actes (fonds du musée des Archives départementales, $\mathrm{n}^{\circ} 99$; divers actes de Pontfraud) que pour les manuscrits (ms. Soissons, bibl. mun. 134 : recueil de sermons en latin comportant des citations en français; ms. Soissons, bibl. mun. 64 : poème satirique; formulaire de testament ajouté à la fin du ms. Laon, bibl. mun. 193).

En ce qui concerne la seconde partie du Moyen Âge, on s'est efforcé constamment de mettre en évidence visuellement les mécanismes d'évolution de l'écriture liés à la cursivité, afin de permettre aux étudiants d'interpréter eux-mêmes les formes qu'ils ne connaissaient pas. Tel ou tel document en écriture de forme a pu servir à la révision des abréviations courantes, mais on s'est concentré, là encore, sur des écritures aussi peu soignées que possible : copies personnelles (ms. Laon, bibl. mun. 146, traité de théologie), fragments additionnels (ms. Rouen, bibl. mun., 399, extraits de bulles pontificales ajoutés à un manuscrit liturgique), documents juridiques ou d'un genre voisin (exercices de transcription tirés du manuel de paléographie d'E. Poulle; extraits du ms. Laon, bibl. mun. 641, dossier consacré à un miracle eucharistique, fin du XVI ${ }^{\mathrm{e}} \mathrm{s}$.).

Pendant la seconde partie de l'année (à partir du mois de janvier) un travail collectif d'édition était en outre proposé aux étudiants volontaires. Il s'agissait d'éditer (à partir de photographies numériques) le chartrier de l'abbaye de Pontfraud, conservé aux archives départementales de l'Yonne. Ce chartrier, comportant des actes du XII ${ }^{\mathrm{e}}$ au $\mathrm{XV}^{\mathrm{e}}$ siècle, a fait l'objet d'une édition (très perfectible) par J. Stein. L'intérêt du dossier est aujourd'hui renforcé par l'existence (inconnue du précédent éditeur) d'un cartulaire du même établissement. Ce travail avait pour but de confronter les étudiants à l'exigeante réalité du travail d'édition : il leur était donc demandé de fournir, pour chaque acte traité, une ébauche de tableau de la tradition, une analyse, une édition critique et une traduction. L'objectif est d'aboutir l'an prochain à la publication en ligne de l'ensemble du dossier, en collaboration avec la section de diplomatique de l'Institut de recherche et d'histoire des textes. Ce projet a donné lieu, lors de séances qui lui étaient consacrées spécifiquement (15 janvier, 5 et 19 mars), ou ponctuellement au début de chaque séance, à des mises au point concernant les principales normes de transcription, les problèmes posés par la chronologie et l'identification des lieux et des personnes. Dans ce cadre ont également été présentés aux étudiants quelques outils d'identification et de chronologie disponibles en ligne (logiciel Millesimo, Corpus Antiphonalium Officii en ligne, etc.). 